\title{
Bariatrics, Metabolic Dysfunction and the Surgeon
}

\author{
Anil Sharma
}

Published online: 30 November 2010

(C) Association of Surgeons of India 2010

Life expectancy has increased over the course of the preceding century. The phenomenon of epidemiological transition reflects the fact that there has been a shift in the major causes of death and disability from communicable, maternal and perinatal causes to chronic, non- communicable ones. The nutritional transition is being witnessed today on a global scale. As a country develops and more people buy processed food rather than growing and buying raw ingredients, an increasing proportion of calories tend to be drawn from sugars added to manufacture food and from relatively cheap oils. Alongside the change in diet, changes in food products and the technology of work and leisure lead to decreases in physical exercise. The consequent epidemic of diet related non-communicable diseases (obesity, diabetes, hypertension and CVD) co-exists with residual under nutrition and is projected to increase rapidly. For example, in India and China a shift in diet towards higher fat and lower carbohydrate is resulting in rapid increases in overweight among all adults in China and mainly among urban residents and high income rural residents in India. Countries which have completed the transition to over nutrition are experiencing a continual increase in levels of obesity, as high fat, high sugar diet and low exercise permeate society.

The evolution of surgical procedures for morbid obesity (bariatric surgery) has been relatively short. Jaw wiring was the first surgical attempt in the modern era to control obesity. Malabsorption, secondary to surgical induction in the form of jegunocolic bypass and subsequently jejunoileal bypass were

\footnotetext{
A. Sharma $(\square)$

Max Institute of Minimal Access, Metabolic \& Bariatric Surgery, Max Healthcare,

Saket,

New Delhi, India

e-mail: Anil.Sharma@maxhealthcare.com
}

performed in obese patients in late 1960's. However, unacceptable complications like electrolyte imbalances, nutrient deficiencies and bacterial overgrowth in the bypassed gut led to a purely malabsorptive approach to bariatric surgery to be abandoned. In 1966, Mason and Ito devised gastric restriction as an alternative conceptual approach to morbid obesity [1]. Various stomach partitioning procedures (gastroplasties) became choice of most bariatric surgeons as they were relatively safe and proved to be generally effective, at least in the short term. In 1979, Mason began performing the gastric bypass, creating a smaller gastric pouch, gastric transection and the use of a jejunal Roux-en-Y limb of various lengths. Gastric banding was first performed during the early 1980's using a fixed band to create a narrow gastric outlet. The current version consists of an inflatable silicon band around the upper stomach, which, connected to a subcutaneous port, allow for adjustments to the band.The next generation of malabsorptive procedures consisted of the biliopancreatic diversion (BPD) developed by Scopinaro [2] which was further notified by Marceau and Hess [3] to include a duodenal switch (DS).

Minimally invasive techniques emerged in bariatric surgery in the early 1990's. Laparoscopic placements of gastric bands were first performed in 1993 and the feasibility of laparoscopic gastric bypass was demonstrated by Wittgrove [4] in 1994. Several studies have demonstrated safety and efficacy of laparoscopic Roux-en-Y gastric bypass since then [5]. The first laparoscopic BPD/DS was performed by Gagner in 1999. Laparoscopic placement of gastric pacing leads has been evaluated in U.S.A. and Europe with limited success. Endoscopic intragastric balloon placement may be used as a primary weight loss procedure in conjunction with dietary modifications but has also been used effectively to produce short term weight loss prior to bariatric and other procedures [6]. 
There was no doubt that bariatric surgery produced substantial and durable weight loss. Sustained weight loss of at least $70 \%$ of excess weight for upto 20 years has been reported from reputed bariatric surgery programs. A reduction in healthcare costs for patients after bariatric surgery has been documented. More importantly, a 50-80\% mortality reduction benefit has been reported with bariatric surgery. These positive results served to firmly establish the credibility of bariatric surgery as a speciality.

As results of bariatric surgery were analyzed, it was apparent that apart from significant and sustained weight loss, there was a marked improvement of deranged metabolic parameters, too. The Swedish obese subjects study scientific group studied lifestyle, diabetes and cardiovascular risk factors 10 years after bariatric surgery. Also 2 and 10 year rates of recovery from diabetes, hypertension and hyperuricemia were more favourable in patients who had bariatric surgery than in the control group. A population based, long term controlled study from the Mayo clinic was reported on the effect of bariatric surgery on the metabolic syndrome in 2008. Roux-en- $Y$ gastric bypass was found to induce considerable and persistent improvement in prevalence of metabolic syndrome.

The most spectacular result, however, in terms of improvement of metabolic dysfunction after bariatric surgery was the resolution of type II diabetes mellitus. In a meta analysis of 22,094 patients, Buchwald et al. [7] found diabetes resolution in $98.9 \%$ of patients undergoing biliopancreatic diversion or duodenal switch, $83.7 \%$ resolution after laparoscopic gastric bypass and $47.9 \%$ after laparoscopic gastric banding. Diabetes control and remission seems to be best obtained with procedures that include an intestinal bypass, as in gastric bypass or biliopancreatic diversion. With a 14 year follow up, Pories et al. [8] found an $83 \%$ resolution rate in 240 obese patients undergoing laparoscopic gastric bypass. Scopinaro et al. [9] reported a $97 \%$ diabetes remission rate in 312 patients maintained at 10 years postoperative follow up. Glycemic control in diabetic patients improves markedly within days of bariatric surgery, in part due to caloric restriction but mainly due to gut peptide (enteroinsular axis) secretion changes. Altered secretion of these gut hormones results in enhanced insulin secretion and sensitivity.

Metabolic surgery is now emerging as a discipline dedicated to the establishment of surgical procedures specifically aimed at treating type II diabetes mellitus and/or severe hypercholesterolemia, and/or other components of metabolic syndrome, independent of BMI. While conventional bariatric surgery seems to treat diabetes in morbidly obese patients, procedures are being evaluated to treat diabetes on its own. Scopinaro insists that metabolic surgery can be considered a discipline if one or more types of operation can be used with the only aim of obtaining, as a result, a metabolic change [10]. Conventional operations, already in practice for morbid obesity include laparoscopic adjustable gastric banding, Roux-en-Y gastric bypass, biliopancreatic diversion with duodenal switch and sleeve gastrectomy. Novel operations aimed specifically towards treatment of diabetes alone are duodeno- jejunal bypass and ileal transposition. The endoluminal duodeno- jejunal bypass sleeve is being evaluated.

Over the decades, there have been occasions where surgeons have been led, perhaps unwittingly, to explore and treat conditions that were traditionally outside the purview of a surgeon. Bariatrics entered the arena of a few enthusiast surgeons in 1960's. The advent of the laparoscopic era witnessed a quantum jump in the number of bariatric surgeons worldwide. Now, metabolic surgery beckons and gastrointestinal surgery is being evaluated as a dedicated treatment for type II diabetes mellitus. This development in metabolic surgery has the potential to attract many more surgeons into its fold. The evolution of minimally invasive bariatric surgery is still in its early stages and there are many opportunities to improve on existing procedures and develop new ones. As new technologies emerge, endoluminal and transgastric procedures will be tested to achieve weight loss with reduced morbidity.

\section{References}

1. Mason EE, Ito C (1967) Gastric bypass in obesity. Surg Clin North Am 47(6):1345-1351

2. Scopinaro N, Gianetta E, Civalleri D, Bonalumi U, Bachi V (1979) Bilio- pancreatic bypass for obesity: II. Initial experience in man. Br J Surg 66:618-620

3. Marceau P, Hould FS, Potvin M, Lebel S, Biron S (1999) Biliopancreatic diversion (duodenal switch procedure). Eur Jgastroenterol Hepatol 11(2):99-103

4. Wittgrove AC, Clark GW, Tremblay LJ (1994) Laparoscopic gastric bypass, Roux-en-Y: preliminary report of five cases. Obes Surg 4(4):353-357

5. De Maria EJ, Sugerman HJ, Kellum JM et al (2002) Results of 281 consecutive total laparoscopic Roux-en-Y gastric bypasses to treat morbid obesity. Ann Surg 235(5):640-645, Discussion 645647

6. Doldi SB, Micheletto G, Di Prisco F et al (2000) Intragastric balloon in obese patients. Obes Surg 10(6):578-581

7. Buchwald H, Avidor Y, Braunwald E, Jensen MD, Pories W, Fahrbach K, Schoelles K (2004) Bariatric surgery a systematic review and meta-analysis. JAMA 292:1724-1737

8. Pories W, Swanson M, MacDonald K, Long SB, Morris PG, Brown BM, Barakat HA, deRamon RA, Israel G, Dolezal GM, Dohm L (1995) Who would have thought it? An operation proves to be the most effective therapy for adult-onset diabetes mellitus. Ann Surg 222:339-352

9. Scopinaro N, Marinan G, Camerini G, Papadia FS, Adami GF (2005) Specific effects of biliopancreatic diversion on the major components of metabolic syndrome: a long-term follow-up study. Diabetes Care 28:2406-2411

10. Scopinaro N, Papadia F, Marinari G, Camerini G, Adami GF (2007) Long term control of type 2 diabetes mellitus and the other major components of the metabolic syndrome after biliopancreatic diversion in patients with BMI $<35 \mathrm{~kg} / \mathrm{m}^{2}$. Obes Surg 17:185-92 